\title{
Diagnostic accuracy of thyroid cytology reporting between a general and a specialist histopathology department, over a five year period
}

\begin{abstract}
Background: According to the current Royal College of Pathologists (RCPath) guidelines, thyroid cytology cases classified as Thy4/Thy5 are expected to be reviewed by a cyto-histopathologist core member of the thyroid multi-disciplinary team (MDT) meeting. We reviewed the usefulness of such practice in a district general hospital $(\mathrm{DGH})$ in the UK.

Methods: Over a five year period, all thyroid fine needle aspirations (FNA) referred from a DGH to a specialist centre with subsequent histology were reviewed. The frequency and degree of discordant thyroid FNA diagnoses between in-house initial diagnosis (ID) and the specialist's second opinion diagnosis (SOD) were analysed, alongside cyto-histological correlations.

Results: A total of 164 cases were reviewed, with $35 \%$ showing diagnostic disagreement between the ID and the SOD. Overall, the SOD was better supported by the subsequent histology, with an improved diagnostic accuracy rate (82\% vs $73 \%)$, sensitivity ( $80 \%$ vs $73 \%)$ and specificity ( $83 \%$ vs $74 \%)$.

Conclusion: This study demonstrates interpretation of thyroid cytology is dependent on the experience of the cyto-histopathologist. Due to the limitations of FNA cytology, the nature of thyroid lesions can be challenging to interpret. A specialist review is considered safe practice to prevent errors and subsequent adverse outcomes in patient management
\end{abstract}

Keywords: thyroid cytology, second opinion diagnosis, diagnostic accuracy
Volume 6 Issue 2 - 2018

\author{
Kanchan Palial,' Derek Roskell, ${ }^{2}$ Gayathri \\ Wathuge' \\ 'Buckinghamshire Healthcare NHS Trust, UK \\ ${ }^{2}$ Oxford University Hospitals NHS Trust, UK
}

Correspondence: Kanchan Palial, Department of

Histopathology, Buckinghamshire Healthcare NHS Trust, UK

Email K.palial@doctors.org.uk

Received: March 06, 2018 | Published: March 29, 2018
Abbreviations: FNA, fine-needle aspiration; ID, initial diagnosis; SOD, second opinion diagnosis; RCPath, Royal College of Pathologists; MDT, multi-disciplinary team; DGH, district general hospital; BTA, British Thyroid Association.

\section{Introduction}

In the United Kingdom (UK), thyroid cytology reporting is based on the British Thyroid Association (BTA)/Royal College of Pathologists (RCPath) Thy classification. This includes Thyl (nondiagnostic), Thy2 (benign), Thy3a (cytological/architectural atypia), Thy3f (suggestive of a follicular neoplasm), Thy4 (suspicious of malignancy) and Thy5 (malignant). This system allows consistent and reproducible reporting of thyroid cytology specimens, and provides a basis for clear communication on which the management of patients with abnormal FNAs can be based. ${ }^{1,2}$

Thyroid cytology can, however, be challenging to interpret due to the nature of thyroid lesions, and a second opinion is often needed for an accurate cytological assessment. According to current RCPath "Guidance on the reporting of thyroid cytology specimens", cases categorised as Thy 4 or Thy 5 are expected to be reviewed by a cyto-histopathology core member of the thyroid MDT. ${ }^{2}$ Prior to this revision, previously published guidance in 2009 recommended that any case classified as Thy3a/f, Thy 4 or Thy 5 should be reviewed by a cyto-histopathologist. Some DGHs routinely refer thyroid cytology cases to a specialist centre in order to comply with these guidelines. Overall, this practice can result in delayed curative surgery, as well as incurring an additional cost. To date, there is limited data on the usefulness of such practice in the UK, whilst the value of second opinions in thyroid cytology has been well investigated elsewhere.

In this study, we compared the in-house initial diagnosis (ID) of thyroid FNAs at Wycombe General Hospital (WGH) with the specialist's second opinion diagnosis (SOD) at the John Radcliffe Hospital (JRH). The frequency and degree of discordant diagnoses between WGH and JRH were reviewed, alongside assessment of histological follow up to help determine whether SOD is beneficial.

\section{Materials and methods}

From 2009 and 2014, all thyroid cytology cases received at WGH were collated using the local institutional database (Winpath). In this study, all referral cases from WGH to JRH for a SOD were included if subsequent histology was available. These cases included an ID of Thy $3 \mathrm{a} / \mathrm{f}$, Thy 4 and Thy 5 and any challenging case that required a specialist review. Cases were excluded if there was an unclear ID or if subsequent histology was not available.

Initially, in each case, the degree of diagnostic agreement/ disagreement between the ID and the SOD was classified into five categories as follows: 
i. Complete diagnostic agreement-Unchanged Thy classification.

ii. Incomplete agreement-Thy3a changed to Thy3f, or vice versa.

iii. Thy category upgraded-Thy category changed from either Thy $3 \mathrm{a} / \mathrm{f}$ to Thy4, Thy4 to Thy 5 or Thy3a/f to Thy5.

iv. Thy category downgraded-Thy category changed from either Thy 5 to Thy4, Thy4 to Thy3a/f or Thy 5 to Thy $3 \mathrm{a} / \mathrm{f}$.

v. Complete diagnostic disagreement-Thy category changed from Thy1 to Thy2, Thy1 to Thy3(a/f)/Thy4/Thy5, Thy2 to Thy3(a/f)/ Thy4/Thy5, or vice versa.

Whilst there are no current widely accepted definitions of false positives, false negatives, true positives or true negatives in the field of thyroid cytology, we defined these terms as follows by correlating the cytological interpretation with the final histological diagnosis (Table 1).

- True negatives (TN), including cytology cases reported as either Thy2 with subsequent benign histology (normal or hyperplastic/ inflammatory process), Thy $3 \mathrm{f}$ with final histology showing a benign follicular neoplasm (adenomatoid nodule, follicular adenoma, hurtle cell adenoma) or Thy3a with subsequent benign histology.

- False negatives (FN), including any case reported as either Thy2 with subsequent malignant histology or Thy $3 \mathrm{f}$ with a non-follicular malignancy on final histology.

- True positives (TP), including Thy3a, Thy4, Thy5 cases with subsequent proven malignant histology. This also includes Thy $3 \mathrm{f}$ where the subsequent histology was either a malignant follicular lesion (including follicular carcinoma and follicular variant papillary carcinoma) or hyalinising trabecular tumour.

- False positives (FP), comprising cases reported as Thy3a, Thy4, Thy5 with subsequent proven normal or benign histology. This included Thy $3 \mathrm{f}$ if the final histology showed a benign process (other than hurtle cell adenoma, follicular adenoma or a cellular adenomatoid nodule)

Table I Definitions of false negative 'FN', false positive 'FP', true positive 'TP' and true negative 'TN' in this study

\begin{tabular}{|c|c|c|}
\hline & $\begin{array}{l}\text { Thy } \\
\text { Classification }\end{array}$ & Histological findings \\
\hline \multirow{3}{*}{ True negative } & Thy 2 & Hyperplastic/inflammatory process \\
\hline & Thy $3 f$ & Benign follicular neoplasm \\
\hline & Thy3a & Benign histology \\
\hline \multirow{2}{*}{ False negative } & Thy2 & Neoplasm (benign or malignant) \\
\hline & Thy3f & Non-follicular malignancy \\
\hline \multirow[t]{2}{*}{ True positive } & $\begin{array}{l}\text { Thy } 3 a, \text { Thy } 4 \text { and } \\
\text { Thy5 }\end{array}$ & Malignant neoplasm \\
\hline & Thy3f & Malignant follicular neoplasm \\
\hline \multirow[t]{2}{*}{ False positive } & $\begin{array}{l}\text { Thy3a/f, Thy } 4 \text { and } \\
\text { Thy5 }\end{array}$ & Hyperplastic/inflammatory process \\
\hline & Thy 4 and Thy 5 & Benign follicular neoplasm \\
\hline
\end{tabular}

Sensitivity $[\mathrm{TP} /(\mathrm{TP}+\mathrm{FN})]$, specificity $[\mathrm{TN} /(\mathrm{TN}+\mathrm{FP})]$, positive predictive value (PPV) $[\mathrm{TP} /(\mathrm{TP}+\mathrm{FP})]$ and overall diagnostic accuracy $[(\mathrm{TP}+\mathrm{TN}) /(\mathrm{TP}+\mathrm{TN}+\mathrm{FP}+\mathrm{FN})]$ were assessed in each center and compared with RCPath guidelines.

\section{Results}

Over five years (2009-2014), 858 thyroid cytology specimens were reported at WGH. Of these, 164 cases were referred from WGH to JRH for a SOD and had subsequent surgical management. The mean patient age was 54 (range 16-88 years) with a predominant number of female patients (88\%). The number of ID and SOD in each Thy category and the correlation between the ID and SOD are demonstrated in figures 1 and 2, respectively.

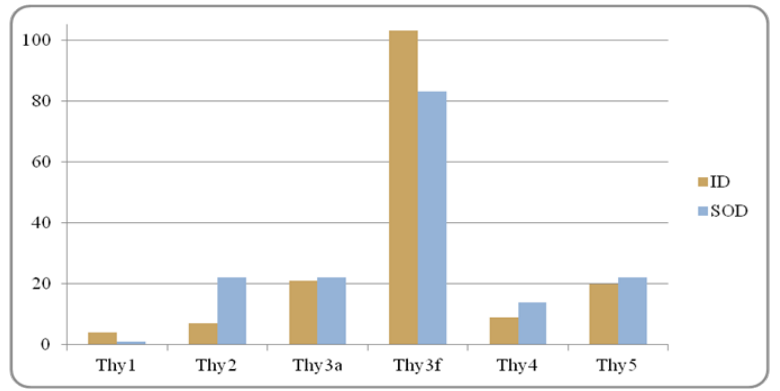

Figure I Number of ID and SOD in each Thy categorisation.

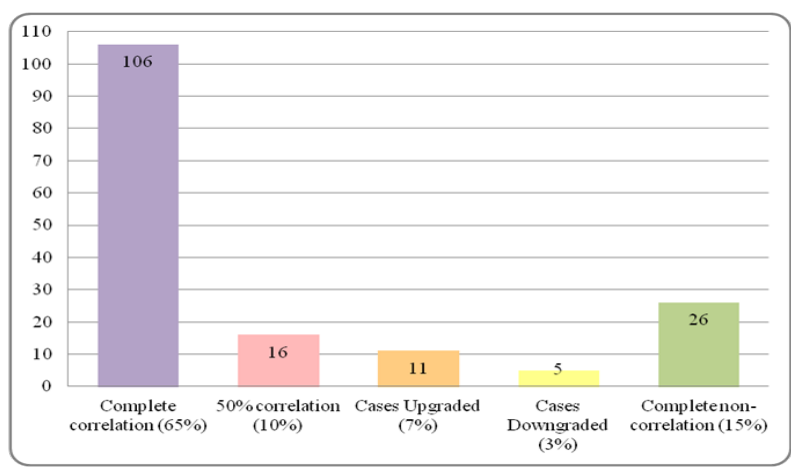

Figure 2 Correlation of Thy Classification between the ID and SOD.

Complete diagnostic agreement between the ID and SOD was seen in 106 out of the 164 cases (65\%). Within this subgroup, most cases showing complete agreement between the ID and SOD were categorised as Thy3f (103 cases were initially referred from WGH with an ID of Thy3f), see Figure 3. Seventeen out of twenty cases referred from WGH with an ID of Thy 5 were completely supported by the SOD and had subsequent histology of papillary thyroid carcinoma. In addition, twelve out of twenty one cases with an ID of Thy3a were completely agreed by the SOD.

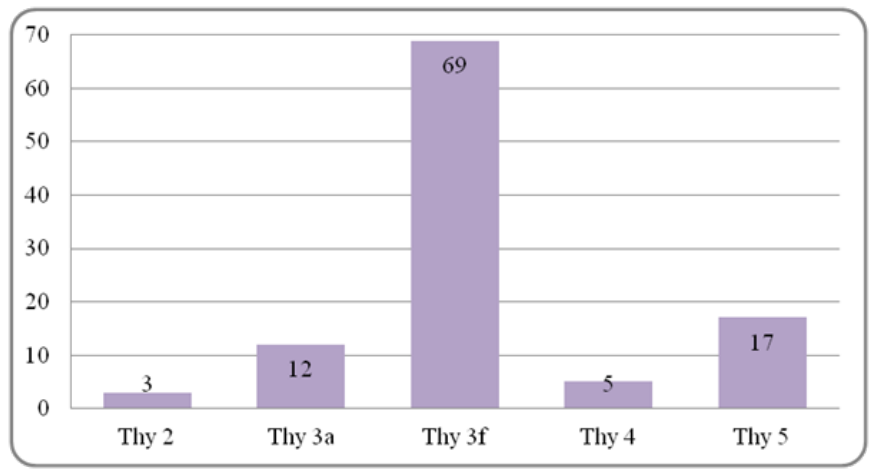

Figure 3 Complete diagnostic agreement between the ID and SOD 
The remaining $35 \%$ of cases in this study (58/164) demonstrated a degree of diagnostic disagreement. Sixteen cases involved partial $(50 \%)$ agreement with seven cases changed from Thy3a to Thy $3 \mathrm{f}$ with the subsequent histology showing follicular adenoma (4/7), Hurtle cell adenoma (2/7) and nodular goitre (1/7). The remaining nine cases were changed from Thy $3 \mathrm{f}$ to Thy $3 \mathrm{a}$ with a final histological diagnosis of papillary thyroid carcinoma (3/9), follicular adenoma $(2 / 9)$, adenomatoid nodule (1/7), colloid nodule (2/7) and parathyroid adenoma (1/7).

A total of eleven cases were upgraded from Thy3a/f to Thy $4(6 / 11)$, Thy3a/f to Thy5 (4/11), and Thy4 to Thy5 (1/11). In 9/11 cases, the final histological diagnosis was papillary thyroid carcinoma, alongside 1/11 hurtle cell adenoma (Thy3f to Thy4) and 1/11 hyalinising trabecular tumour (Thy3f to Thy4).

Five cases were downgraded, including two cases downgraded from Thy5 to Thy4 with the histological diagnosis in each case being papillary thyroid carcinoma. Two cases were appropriately downgraded from Thy 4 to Thy $3 \mathrm{f}$ with subsequent histology showing follicular adenomas. One case was downgraded from Thy4 to Thy $3 \mathrm{f}$ with subsequent histology showing multinodular goitre.

Twenty six cases showed complete diagnostic disagreement, see Figure 4. This included eighteen cases which were appropriately changed from Thy3f (ID) to Thy2 (SOD), with final histological diagnoses including nodular goitre (12/18), Hashimoto's thyroiditis $(2 / 18)$ and colloid nodule $(2 / 18)$. Three cases were changed from Thy2 to Thy 3 , with subsequent histology showing follicular adenoma $(1 / 3)$, follicular adenoma and papillary thyroid carcinoma (1/3) and multinodular goitre with dominant nodule (1/3). Two cases were changed from Thy 1 to Thy3f, including a follicular adenoma $(1 / 2)$ and Hashimoto's Thyroiditis (1/2). A further single case was changed from Thy1 to Thy2 (benign colloid nodule), Thy1 to Thy4 (papillary thyroid carcinoma) and Thy5 to Thy1 (nodular goitre).

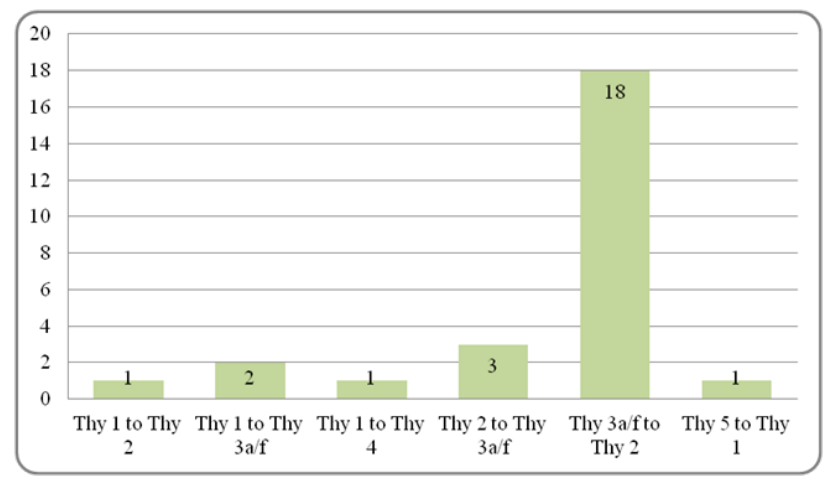

Figure 4 Complete non-correlation between the ID and SOD.

Analysis of diagnostic accuracy of detecting thyroid malignancy on cytology by the ID and SOD is displayed in Table 2. Overall, the ID false positive rate in this study was $26 \%$, with the majority of constituent cases initially classified as Thy $3 \mathrm{f}$. The specialist review avoided eleven false positive cases, where the final histological diagnosis was either Hashimoto's thyroiditis or multinodular goitre, improving the false positive rate to $17 \%$.

The false negative rate of the ID was $27 \%$, with all fifteen false negatives initially classified as Thy $3 \mathrm{f}$ or Thy 2 and subsequent histology revealing papillary thyroid carcinoma. The SOD avoided eight of these false negative cases with re-classification to Thy $3 \mathrm{a}-$ Thy5. In contrast, the second review introduced five false negatives, with each case downgraded from Thy3f to Thy2, and subsequent histology showing a follicular neoplastic lesion. However, overall, the SOD false negative rate was lower than the ID at $20 \%$.

Table 2 Diagnostic accuracy of thyroid cytology reporting by ID and SOD, with comparison with RCPath guidelines

\begin{tabular}{llll}
\hline & ID & SOD & $\begin{array}{l}\text { RCPath } \\
\text { Recommended Range }\end{array}$ \\
\hline False positive rate & $26 \%$ & $17 \%$ & $0-5.7 \%$ \\
False negative rate & $27 \%$ & $20 \%$ & $0-5 \%$ \\
Sensitivity & $73 \%$ & $80 \%$ & $65 \%-98 \%$ \\
Specificity & $74 \%$ & $83 \%$ & $76-100 \%$ \\
PPV for Thy3a & $100 \%$ & $100 \%$ & $17 \%$ \\
PPV for Thy3f & $26 \%$ & $36 \%$ & Up to $40 \%$ \\
PPV for Thy4 & $100 \%$ & $92 \%$ & Up to $68 \%$ \\
PPV for Thy5 & $95 \%$ & $100 \%$ & Up to $100 \%$ \\
Overall accuracy & $73 \%$ & $82 \%$ & $69-97 \%$
\end{tabular}

Following cyto-histological correlation, we found that the sensitivity in the ID and SOD was $73 \%$ and $80 \%$, respectively. However, the specificity in the ID was $74 \%$, which is below the recommended RCPath range (76-100\%), whilst it was much higher by the SOD at $83 \%$. The PPV in both centers was within the recommended ranges for Thy $3 a$, Thy $3 f$, Thy 4 and Thy5. In particular, the PPV for Thy 5 by the ID and SOD was $95 \%$ and $100 \%$, respectively.

\section{Discussion}

Thyroid cytology provides both an accurate and cost effective test to deliver a diagnosis of a thyroid neoplasm, and allows the patient to be triaged for either conservative or surgical management. ${ }^{3,4}$ Misinterpretation of thyroid cytology can have a significant impact on patient management, as well as carrying the prospect of litigation. ${ }^{5}$ SOD in thyroid cytology has shown to improve cytological interpretation and to prevent mismanagement. In this study, we investigated the value of SOD in thyroid cytology.

The majority of cases in this study demonstrated complete agreement in the Thy classification between the ID and SOD (65\%), with highest concordance in Thy $3 \mathrm{f}$ cases. The remainder of cases showed a degree of diagnostic disagreement (35\%). Similar results reported by Park et al showed a diagnostic agreement rate of $74 \%$ between the ID and SOD using the Bethesda system for Thy categorisation, alongside a diagnostic disagreement rate of $26 \%{ }^{6}$ Whereas, Tan et al reported a much lower discordant rate of $18 \%$, and among these surgical management was changed for $30 \%$ of patients. ${ }^{7}$

In this study, $15 \%$ of cases showed complete diagnostic disagreement with most cases appropriately changed from Thy $3 \mathrm{f}$ (ID) to Thy2 (SOD). Of the cases showing partial disagreement, $85 \%$ of cases changed from Thy 3 a to Thy 3 f were supported by the histological follow up. However, in only $33 \%$ of cases changed from Thy3f to Thy3a, subsequent histological findings of malignancy were present. This gives further evidence that management of Thy $3 \mathrm{a}$ 
cytology cases should be very careful with vigilant correlation with the clinical features and radiological findings. ${ }^{8}$

$80 \%$ of the "upgraded" cases, with SOD of either Thy 4 or Thy 5 had subsequent histology of papillary thyroid carcinoma. In addition, three out of five cases downgraded from Thy 4 to Thy 3 included cases of two follicular adenomas and one multinodular goitre. By improving the false negative rate and false positive rate, respectively, a SOD has shown to prevent cytological misinterpretation and potential adverse patient management. However, the direct change in management was difficult to record in this study, as surgical excision is not just dependent on the Thy categorisation, but also by other factors including patient age, size of the lesion and multifocality.

Literature on malignancy detection on thyroid cytology indicates sensitivity ranges between $65 \%$ to $98 \%$, specificity of $76-100 \%$, false-negative rate of $0-5 \%$ and false positive rate of $0-5.7 \% .^{2}$ The specificity for detecting thyroid malignancy by the ID was found to be slightly lower at $74 \%$, whilst the sensitivity and specificity in the SOD were within the recommended ranges.

The false positive and false negative rates at both centers were higher than the published data stated in the RCPath "Guidance on the reporting of thyroid cytology specimens". However, this document acknowledges the limitations of the comparison with international data due to how results are categorized and analysed. ${ }^{2}$ Moreover, the definitions for FP, TP, FN and TN in thyroid cytology reporting are not outlined in the RCPath guidance, and our definitions may vary to those used elsewhere. Causes for false negative rates in thyroid cytology could be explained by undersampling of lesions, or sampling of cystic tumors or lesions where there maybe dual pathology. ${ }^{9}$ Since thyroid lesions can have variable morphology in appearance, it is important to ensure adequate sampling to provide a representative specimen for analysis. In particular, this study included three cases demonstrating dual pathology, in which one component in each case being papillary thyroid carcinoma that was not suspected on the initial FNA. This shows that interpretation of thyroid cytology is not only dependent on the experience of the cytopathologist, but also the experience of the radiologist and aspirator. In addition, this study further supports that Hashimoto's thyroiditis can introduce both false positives and false negatives in thyroid cytology, as seen in previous studies. ${ }^{10,11}$ This is mainly due to the considerable morphological overlap with malignant cells seen in papillary thyroid carcinoma.

The overall diagnostic accuracy of detecting malignancy in thyroid cytology ranges between $69-97 \%$, as mentioned in current RCPath guidance. ${ }^{2}$ This study has revealed that diagnostic accuracy in the ID was $73 \%$ (within recommended range), whilst this was much higher in in the SOD at $82 \%$. In addition, we have demonstrated that a SOD in thyroid cytology is associated with an improved sensitivity, specificity, PPV and diagnostic accuracy in detecting malignancy.

\section{Conclusion}

In this study, we reviewed the usefulness of a SOD in thyroid cytology in the UK. With improved overall diagnostic accuracy by the SOD by $9 \%$, we demonstrate that thyroid cytology interpretation is dependent on the experience of the cyto-pathologist. We therefore further support that a SOD is considered to be safe practice, by providing an important tool to detect interpretive errors which may significantly impact on patient management.

\section{Acknowledgments}

None.

\section{Conflicts of interest}

No potential conflicts of interest relevant to this article were reported.

\section{References}

1. Lobo C, McQueen A, Beale T, et al. The UK Royal College of Pathologists thyroid fine-needle aspiration diagnostic classification is a robust tool for the clinical management of abnormal thyroid nodules. Acta Cytol. 2011;55(6):499-506.

2. Royal collage of pathologists. Guidance on reporting thyroid cytology specimens. Version no 2, United Kingdom; 2016.

3. Gerhard R, Boerner SL. Evaluation of indeterminate thyroid cytology by second-opinion diagnosis or repeat fine-needle aspiration: which is the best approach?. Acta Cytol. 2015;59(1):43-50.

4. Yang J, Schnadig V, Logrono R, et al. Fine-needle aspiration of thyroid nodules: a study of 4703 patients with histologic and clinical correlations. Cancer. 2007;111(5):306-315.

5. Hamady ZZ, Mather N, Lansdown MR, et al. Surgical pathological second opinion in thyroid malignancy: impact on patients' management and prognosis. Eur J Surg Oncol. 2005;31(1):74-77.

6. Park JH, Kim HK, Kang SW, et al. Second opinion in thyroid fine-needle aspiration biopsy by the Bethesda system. Endocr J. 2012;59(3):205212.

7. Tan YY, Kebebew E, Reiff E, et al. Does routine consultation of thyroid fine-needle aspiration cytology change surgical management? J Am Coll Surg. 2007;205(1):8-12.

8. Brophy C, Mehanna R, McCarthy J, et al. Outcome of Subclassification of Indeterminate (Thy-3) Thyroid Cytology into Thy-3a and Thy-3f. Eur Thyroid J. 2015;4(4):246-251.

9. Onal ED, Saglam F, Sacikara M, et al. The diagnostic accuracy of thyroid nodule fine-needle aspiration cytology following thyroid surgery: a case-control study. Endocr Pathol. 2014;25(3):297-301.

10. Olson MT, Boonyaarunnate T, Aragon Han P, et al. A tertiary center's experience with second review of 3885 thyroid cytopathology specimens. J Clin Endocrinol Metab. 2013;98(4):1450-1457.

11. Mahajan A, Lin X, Nayar R. Thyroid Bethesda reporting category, 'suspicious for papillary thyroid carcinoma', pitfalls and clues to optimize the use of this category. Cytopathology. 2013;24(2):85-91. 